\title{
Distorted body representations are robust to differences in experimental instructions
}

\author{
Luigi Tamè $^{1}$ - Nicola Bumpus ${ }^{1} \cdot$ Sally A. Linkenauger $^{2} \cdot$ Matthew R. Longo $^{1}$
}

Published online: 15 February 2017

(C) The Psychonomic Society, Inc. 2017

\begin{abstract}
Several recent reports have shown that even healthy adults maintain highly distorted representations of the size and shape of their body. These distortions have been shown to be highly consistent across different study designs and dependent measures. However, previous studies have found that visual judgments of size can be modulated by the experimental instructions used, for example, by asking for judgments of the participant's subjective experience of stimulus size (i.e., apparent instructions) versus judgments of actual stimulus properties (i.e., objective instructions). Previous studies investigating internal body representations have relied exclusively on 'apparent' instructions. Here, we investigated whether apparent versus objective instructions modulate findings of distorted body representations underlying position sense (Exp. 1), tactile distance perception (Exp. 2), as well as the conscious body image (Exp. 3). Our results replicate the characteristic distortions previously reported for each of these tasks and further show that these distortions are not affected by instruction type (i.e., apparent vs. objective). These results show that the distortions measured with these paradigms are robust to differences in instructions and do not reflect a dissociation between perception and belief.
\end{abstract}

Keywords Body parts · Instructions judgment size · Distortions body representations

Luigi Tamè

luigi.tame@gmail.com

Matthew R. Longo

m.longo@bbk.ac.uk

1 Department of Psychological Sciences, Birkbeck, University of London, WC1E 7HX London, UK

2 Department of Psychology, Lancaster University, Lancaster, UK
Distortions and misperceptions of the body are a conspicuous feature of numerous serious clinical disorders, including psychiatric conditions such as eating disorders (Treasure, Claudino, \& Zucker, 2010) and body dysmorphic disorder (Phillips, 2005), as well as neurological conditions such as phantom limbs (Ramachandran \& Hirstein, 1998), somatoparaphrenia (Vallar \& Ronchi, 2009), and xenomelia (Brugger, Lenggenhager, \& Giummarra, 2013). Such conditions have fascinated researchers and the wider public, not only because of their clinical significance but also on account of the striking conflict they present to the intimate and apparent experience we have of our own body with respect to how it really is physically. Recently, a growing literature has started to show that distorted body representations are not unique to disease, but are a characteristic part of healthy perception/cognition. Indeed, numerous studies have revealed large and highly consistent distortions of body representations in healthy human adults (Fuentes, Longo, \& Haggard, 2013; Hach \& Schütz-Bosbach, 2010; Linkenauger, Wong, et al., 2014; Linkenauger, Witt, Bakdash, Stefanucci, \& Proffitt, 2009; Longo \& Haggard, 2010, 2011, 2012; Saulton, Dodds, Bülthoff, \& de la Rosa, 2015).

\section{Distorted body representations in healthy adults}

One series of studies has investigated distorted body representations mediating position sense. While several forms of afferent signals from joints, the skin, and muscles provide information about the posture of our body (Proske \& Gandevia, 2012), no afferent signal provides information about body size and shape. To perceive the spatial location of a body part, however, information about the angles of joints needs to be combined with information about the length of segments connecting joints, which many researchers claim comes from a stored body model 
(Longo, Azañón, \& Haggard, 2010). Longo and Haggard (2010) developed a paradigm to isolate and measure this body model. Participants sat with their left hand on a table, with the dorsum facing up and their hand flat and fingers completely straight. They used a long baton to judge the location of the knuckle and tip of each finger of their occluded hand. These landmarks were the centre of the knuckle at the base of each finger and each fingertip. On each trial, participants were verbally instructed which landmark to judge. Participants were instructed to take their time, be precise, avoid ballistic pointing, and avoid strategies such as tracing the outline of the hand. Before each trial, participants moved the tip of the baton to a blue dot at the edge of the board. By comparing the relative location of each landmark, they constructed implicit maps of represented hand shape, which could then be compared to actual hand shape. These maps were drastically distorted, in a highly consistent manner across individuals. Specifically, across a number of studies, three characteristic patterns of distortions are apparent: underestimation of finger length (i.e., the distance between the knuckles and tip of the fingers), overestimation of the hand width (i.e., distance between pairs of knuckles), and a radial-ulnar gradient with underestimation of finger length increasing systematically from the thumb to the little finger (Ferrè, Vagnoni, \& Haggard, 2013; Longo, 2014; Longo \& Haggard, 2010, 2012; Longo, Long, \& Haggard, 2012; Lopez, Schreyer, Preuss, \& Mast, 2012; Mattioni \& Longo, 2014).

Another series of studies investigated distorted body representations using tactile size perception. For instance, Longo and Haggard (2011) used a modified version of the classical Weber's illusion paradigm (Weber, 1834/1996), in which the perceived distance between two points touching the skin increases as the points are moved from a region of low tactile sensitivity to one of higher sensitivity. However, rather than comparing perceived tactile distance on two different skin surfaces, Longo and Haggard investigated the shape of the body by measuring perceived tactile distance in different orientations on a single skin surface (i.e., the back of the hand). The rationale was that distortion of body shape should produce an anisotropy in perceived size of tactile objects as a function of orientation. Specifically, if the hand is represented as being longer and thinner than it really is, distances oriented proximodistally, along the body surface, should feel larger than those oriented mediolaterally, across the width of the body. Conversely, if the hand is represented as being squatter and wider than it actually is, distances oriented across the hand should be perceived as larger than those oriented along the hand. Longo and Haggard (2011) found that tactile distances on the dorsum of the hand were perceived as approximately $30 \%-40 \%$ larger when they were oriented mediolaterally (across the hand) than proximally (along the hand), demonstrating the presence of anisotropy on the hand dorsum with a clear bias for tactile distances to be perceived as larger when oriented mediolaterally. Several other recent studies have reported similar results (Canzoneri et al., 2013; Longo \& Sadibolova, 2013). Similar anisotropies have also been shown to be present when tactile stimuli are presented on other body parts, such as the forearm and leg (Green, 1982).

Other distortions are also present when participants estimate the perceived length of body parts using visual matching tasks. For example, Linkenauger and colleagues (Linkenauger et al., 2009) asked participants to indicate the perceived length of their right and left arms by adjusting a blank tape measure so that the length of the tape measure matched the perceived length of their arm. Right-handed participants judged their right arm as longer than their left arm, though there was actually no difference in length between the two. Similarly, Linkenauger and colleagues (Linkenauger, Wong, et al., 2014) used a visual estimation task in which participants were asked to measure the length of one body part using another body part (e.g., how many hand lengths would be required to measure one's arm). They found that participants showed systematic distortions in the perception of bodily proportions that were a function of each body part's relative tactile sensitivity and physical size. Finally, Longo and Haggard (2012) used a visual comparison task to obtain estimates of perceived finger length. They found clear underestimation of finger length analogous to those they had previously found for position sense (Longo \& Haggard, 2010), though smaller in magnitude.

\section{Apparent and objective instructions}

In the late $1800 \mathrm{~s}$, Titchener coined the term 'stimulus-error' to refer to the 'objectification' of the responses given by the participants as a source of error (Titchener, 1909). Already in these early years of the psychological studies, psychologists recognised the presence and possibly the problems that may derive from the different attitudes that may be used by participants to give their reports about a certain psychological experience (for a discussion on this issue, see Boring, 1921, and more recently, Chirimuuta, 2016).

A common feature of the studies we described in the previous section is the type of instruction given to participants. In particular, participants have generally been asked to base their responses on their subjective feelings of body size, body location, or stimulus size (i.e., apparent prospective). This raises the possibility of a dissociation between participants' subjective feelings and their actual beliefs (i.e., objective prospective). That is, they may realise that their responses are inaccurate, yet nevertheless respond - as instructed - based on their subjective feeling. For example, a participant performing Longo and Haggard's (2010) hand localisation task might reason that 'I know the tip of my index finger is over there, but it feels like it is right here." Similarly, a participant performing Linkenauger and colleagues' (2009) arm length estimation task might reason that 'I know my left arm is a bit longer than that, but it feels this 
long." In all these previous studies, participants, implicitly or explicitly, were always instructed to perform the task using an 'apparent' prospective. The rational of this approach was dictated by the fact that these investigators were interested in participants' judgements of their feeling of the position or size of the body - in other words, in the participant's perception of the spatial position of their tip of the finger or the length of their arm. This type of instruction, focusing on the participant's subjective experience, is known as an 'apparent' instruction (Carlson, 1977). Apparent instructions are thought to direct the observers to base their judgments on more perceptual sources of spatial information (Predebon, 1992). On the contrary, participants can be instructed to perform a task assuming an 'objective' perspective, in which the focus is on the participant's actual beliefs about the stimulus. For example, a participant performing the localisation task of Longo and Haggard (2010) could be asked to judge the location where they really think the tip of their fingertip is, regardless of their subjective experience. This type of approach is thought to direct the observers to base their judgments on more cognitive sources of spatial information where a sort of correction from the subjective feelings is supposed to be necessary (Predebon, 1992). Cognitive sources of information might reflect the participants' memory of the characteristics of object size (e.g., a hand) or their actual beliefs about the relevant size-distance relations.

A large literature in visual psychophysics has shown that the type of instruction given to participants can have dramatic effects on the pattern of data produced. Indeed, Leibowitz and Harvey (1969) have shown that the most effective experimental variable in size-constancy experiments is the instruction given to the participants. In the visual modality, it has been shown that the judged size of a familiar object (e.g., a playing card) remains constant under objective (participants' actual beliefs) instructions, but decreases under apparent (participants' subjective feelings) instructions as the physical distance to the object increased, resulting in a decrease in the size of the object's retinal projection (Gogel \& Da Silva, 1987). Similarly, Predebon (1980) asked participants to judge the length of different body parts (i.e., forearm, head, foot, hand) and objects (brown planar cardboard figures similar in shape to the body part stimuli) that were visually presented in front of the participant, using apparent and objective instructions. He found that spatial judgments of body stimuli differ systematically from spatial judgments of nonbody stimuli (Predebon, 1980).

A clear example of the two attitudes in the context of body representations in the tactile domain is provided by the Aristotle illusion (Benedetti, 1985). When people cross the index and middle fingers and touch their nose between the two fingers, they experience the feeling two noses. The apparent instruction would be 'How many noses did you perceive?'; whereas, an objective question would be 'How many noses do you believe that you have?' In the former case, people experiencing the illusion would respond 'two'; whereas, in the latter case, they would respond 'one'. This is a clear example in which the 'apparent' and 'objective' judgments about the body can be clearly dissociated. Similarly, in the case of phantom limbs following amputation, the patient experiences the limb continuing to exist, but knows perfectly well that it does not. Likewise, participants experiencing the rubber hand illusion (Botvinick \& Cohen, 1998) report feeling like the rubber hand is part of their body, but of course do not actually believe that it is.

Recently, Ekroll and colleagues (Ekroll, Sayim, Van der Hallen, \& Wagemans, 2016), using a visuo-proprioceptive illusion (the 'shrunken finger illusion'), reported a dissociation between apparent and objective instructions in a body-size estimation judgment. They asked participants to estimate the length of a finger (i.e., middle finger), viewing it directly from above when a semi-spherical coloured or a transparent shell was placed on the top of it, depending on the condition. The semispherical coloured shell creates the illusory feeling of a complete ball that in turn produced the bodily experience of a shorter finger. In one experiment, participants assumed different approaches to the task, namely it was asked using a stick to point (1) where they think the tip of their finger was located (a cognitive judgment) and (2) where they feel the tip of their finger was located (a perceptual judgment). Critically, finger's length underestimation was significantly larger (i.e., about 4 times) when a perceptual rather than a cognitive approach was adopted (apparent vs. objective). Ekroll and colleagues interpreted this result as evidence that the illusory experience of a 'shrunk' finger maybe based on perceptual rather than cognitive processing.

In the same way, participants' response attitude has been shown to affect the judgments on body-size perception, not only in healthy subjects but also in clinical populations. For instance, distortions in the body size estimates have been shown to be present in patients with eating disorders (Cash \& Deagle, 1997; Slade \& Russell, 1973), and it has been hypothesized to originate from either a veridical experience of an altered internal representation of the body or by purely attitudinal bias with no perceptual base (Ben-Tovim, Walker, Murray, \& Chin, 1990). Specifically, Ben-Tovim and colleagues argue that, if the bias of body size estimation showed in people with eating disorders reflects an attitudinal bias towards the body (e.g., feeling that the body is too fat), it could be that patients feel fat, but do not really believe that they are (Ben-Tovim et al., 1990). Therefore, in both healthy subjects and patients, it remains unclear whether the type of instruction provided to the participant can affect the judged size and shape of their body. However, the type of instruction may clarify whether these distortions are perceptual or cognitive in nature.

\section{The present study}

The current study investigated the use of both apparent and objective instructions in three tasks that have recently been found to 
reveal large and highly consistent distortions of the estimated size and shape of the hand. In each case, previous studies have used only apparent instructions. The key question is whether the distortions will be reduced or eliminated when participants are given objective instructions. If there is a dissociation between subjective feelings and actual beliefs or even possibly the operation of demand characteristics on participants' judgments, then distortions should be reduced for objective instructions, compared to apparent instructions. By contrast, if there is not a dissociation between subjective feelings and actual beliefs on participants' judgments, then distortions should not be modulated by the type of instruction. This latter scenario would further support the interpretation that these misrepresentations reflect distorted underlying body representations. The rationale of the current study is to investigate whether the judgements about the body are, as for any other object, susceptible to different types of instructions. The aims of this research were twofold: first, we aimed to determine whether the distorted representations of the body we have previously reported (e.g., Longo \& Haggard, 2010, 2011) might, at least in part, reflect a dissociation between feeling and belief; second, given the clear evidence for dissociations between the objective and apparent attitudes in studies of visual object perception, we were interested in revealing whether body representations show similar effects.

In Experiment 1, we used the 'psychomorphometric' paradigm in which participants indicate the perceived location of landmarks on their occluded hand (Longo \& Haggard, 2010). By comparing the relative locations of each landmark, a perceptual map of hand size and shape can be constructed and compared against actual hand structure. In Experiment 2, we measured anisotropy of tactile size perception on the back of the hand (Longo \& Haggard, 2011). On each trial, two pairs of touches defining different tactile distances were applied sequentially to the hand, one pair oriented proximodistally (along the hand) and the other oriented mediolaterally (across the hand). Participants made two-alternative forced choice (2AFC) judgments of which distance felt larger. In Experiment 3, we used a more explicit measure of body image in which participants adjusted the length of lines presented on a screen to match the length of different parts of their hand (Longo \& Haggard, 2012).

\section{Experiment 1: A 'psychomorphometric' investigation of implicit body representation under different instructions}

\section{Method}

Participants Twenty-six participants (mean $\pm S D=28.5 \pm$ 11.9 years; 17 females) took part in the study. Participants of all experiments reported normal or corrected-to-normal vision and touch. All participants in all experiments gave their informed consent prior to participation. The study was approved by the local ethics panel. Participants, except for one, were all right-hand, as assessed by the Edinburgh Handedness Inventory (Oldfield, 1971; $M=77$, range: $-100-100$ ).

Design In these series of experiments, we decided to adopt a within-subject design, in which the same participants perform the task in all conditions. ${ }^{1}$

Procedure Procedures for this task were similar to those we have used previously (Longo, 2014; Longo \& Haggard, 2010, 2012; Longo et al., 2012). Participants placed their left hand palm-down on a table, aligned with their body midline, the hand flat and fingers straight. A board $(40 \times 40 \mathrm{~cm})$ rested on four pillars (6-cm high) and occluded the hand. A webcam suspended $27 \mathrm{~cm}$ above the occluding board captured photographs $(1600 \times 1200$ pixels $)$ controlled by a custom MATLAB (MathWorks, Natick, MA) script. Participants used a long baton (35-cm length; 2-mm diameter) to indicate the perceived location of different landmarks on their occluded left hand. Ten landmarks were used: the knuckles (i.e., centre of the knuckle at the base of each finger) and tips (i.e., most distal point) of each finger.

On each trial, participants were verbally instructed which landmark to localise. They were instructed to be precise and avoid ballistic pointing or strategies such as tracing the outline of the hand. To ensure independent responses, participants moved the baton to the side of the table between trials. When the participant indicated their response, a photograph was taken and stored for offline coding. In different blocks of trials, the experimenter asked participants to adopt different approaches to the task. At the beginning of the study, the following description of the two approaches was given:

In different blocks of trials, I'll ask you to adopt different approaches to the task. On some blocks, I'll ask you to judge the location where it FEELS like each part of your hand is located. In this case, we're interested in your subjective experience of the location of your hand. On other blocks, I'll ask you to judge the location where

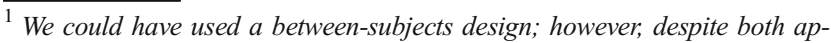
proaches having some benefits and disadvantages, our evaluation is that, for our purposes, a within-subjects design is the most appropriate. It is likely that a between-subjects design in this specific context would be problematic since it would have raised severe concerns that the manipulation of instructions is simply too subtle to be detected. Instead, we aimed to make the distinction between apparent' and 'objective' instruction conditions as clear and salient as possible. Although by using a within-subjects design, participants may try to produce similar patterns in the two conditions; this possibility is very unlikely because the tasks we use are nontransparent in the sense that participants have no idea about the specific patterns of distortion in their data (see previous studies Longo \& Haggard, 2010, 2011). If they do not realize that they are producing a certain pattern (e.g., squat, fat hand map), then it is not obvious how they could deliberately produce equally squat, fat hand maps in the both conditions. Finally, a recent report by Ekroll and colleagues (2016) successfully adopted a within-subject design in a very similar manipulation.
} 
you think each part of your hand REALLY IS. In this case, we're interested in your objective beliefs about the location of your body.

There were two blocks of 30 trials for each instruction condition. Apparent and objective blocks were presented counterbalanced in ABBA sequence, with the first condition counterbalanced across participants. Each block included three miniblocks of one trial of each landmark in random order. At the very beginning and end of each block, a photograph was taken without the occluder showing the participant's hand. This allowed measurement of true hand proportions as well as a check that the hand hadn't moved during the course of the block. To facilitate coding, a black mark was made on each knuckle with a felt pen. A 10 -cm ruler appeared in the photographs without the occluder, allowing conversion between pixels and $\mathrm{cm}$.

Analysis Analysis methods were similar to our previous studies with this paradigm (Longo \& Haggard, 2010, 2012a). Pixel coordinates of landmarks were coded and averaged, resulting in one map for each block. Distances were calculated between the tip of each finger and its knuckle and between the knuckles of finger pairs. The overestimation values of fingers length and the underestimation values of hand width were entered into two separate two-way analysis of variance (ANOVAs), with FINGER (thumb, index finger, middle finger, ring finger, little finger) and INSTRUCTION (apparent, objective) as within-participant factors. Two-tailed $t$ tests were used for all planned comparisons.

For visual comparison of actual and represented hand shape for the apparent and objective instruction conditions, we used generalised Procrustes analysis (GPA). GPA aligns sets of homologous landmarks, removing differences in location, rotation, and scale, isolating differences in shape (Bookstein, 1991). Before GPA, fingers were rotated to a common set of angles to remove postural differences. GPA was conducted using Shape (a MATLAB toolbox from Dr Simon Preston, freely available from download [https://www.maths.nottingham.ac.uk/personal/spp/ shape.php] based on an algorithm originating from Gower, 1975; Ten Berge, 1977).

Finally, the overall aspect ratio of the hand was quantified using Napier's (1980) shape index, a ratio of hand width to length. As in previous studies (Longo, 2014; Longo \& Haggard, 2010; Mattioni \& Longo, 2014), the distance between the knuckles of the index and little fingers was taken as a measure of hand width, and the length of the middle finger as a measure of hand length. The shape index is calculated as: $\mathrm{SI}=100 \times$ (width/length). Large shape indices indicate a squat, wide hand, while small values indicate a thin, slender hand. The shape index was calculated for each participant for her or his actual hand, as well as for the hand maps in each condition.

\section{Results and discussion}

Previous studies using the localization task (Longo, 2014; Longo \& Haggard, 2010, 2012; Longo et al., 2012) have shown large distortions of the implicit body representation of the hand, including (1) overall underestimation of finger length, (2) a radial-ulnar gradient with underestimation increasing from the thumb to the little finger, and (3) overestimation of hand width. All of these biases were apparent in the present study in both the apparent and objective conditions. As shown in Fig. 1a, averaging across the five fingers, there was clear underestimation of the finger length in both the apparent $(M \pm S E=-27.8 \% \pm 3.9), t(25)=-8.91, p<.0001$, $d=1.75$, and objective $(M \pm S E=-30.7 \% \pm 3.6), t(25)=-10.31$, $p<.0001, d=2.02$, instruction conditions. There was no significant difference in the amount of underestimation between the two conditions, $t(25)=1.55, p=.13, d_{\mathrm{z}}=0.30$. Underestimations of fingers length for the apparent and objective conditions were strongly correlated, $r(25)=.822, p<$ .0001 .

To assess the gradient of fingers' length underestimation across fingers, least-squares regression was used to estimate the change in underestimation for a shift of one digit towards the little finger. In agreement with previous reports, we found a clear underestimation from the thumb to little finger in both the apparent (mean $\beta=4.6 \% /$ finger), $t(25)=-5.19, p<.0001$, $d=1.02$, and objective (mean $\beta=5.1 \% /$ finger), $t(25)=-10.17$, $p<.0001, d=1.99$, instruction conditions. The magnitude of this gradient did not differ between the apparent and objective instructions, $t(25)=0.61, p=.55, d_{\mathrm{z}}=0.12$. Again, there was a significant correlation between slopes in the two conditions, $r(25)=.550, p=.004$. These results provide clear evidence that the distorted estimations of finger length are not affected by the type of instruction, apparent or objective, provided to the participants.

As shown in Fig. 1b, there was also clear overestimation of the distance between pairs of knuckles. Taking the distance between the knuckles of the index and little fingers as an overall measure of the hand width, there was clear overestimation for both the apparent $(M \pm S E=64.8 \% \pm 6.5), t(25)=$ $9.92, p<.0001, d=1.94$, and objective $(M \pm S E=65.4 \%$ $\pm 5.1), t(25)=12.80, p<.0001, d=2.51$, conditions. The magnitude of overestimation did not differ between the two conditions, $t(25)=-0.15, p=.88, d_{\mathrm{z}}=0.03$. However, the hand width measures for the apparent and objective instruction conditions (i.e., distance between the knuckles of the index and little fingers) were strongly correlated, $r(25)=$ $.788, p<.0001$.

The shape indices for hand maps were greater than for the actual hand for both the apparent (144.9 vs. 56.7), $t(25)=$ 11.04, $p<.0001, d=2.17$, and objective (153.5 vs. 56.7 ), $t(25)=8.69, p<.0001, d=1.70$, instructions. The shape index for the two experimental conditions did not differ significantly 


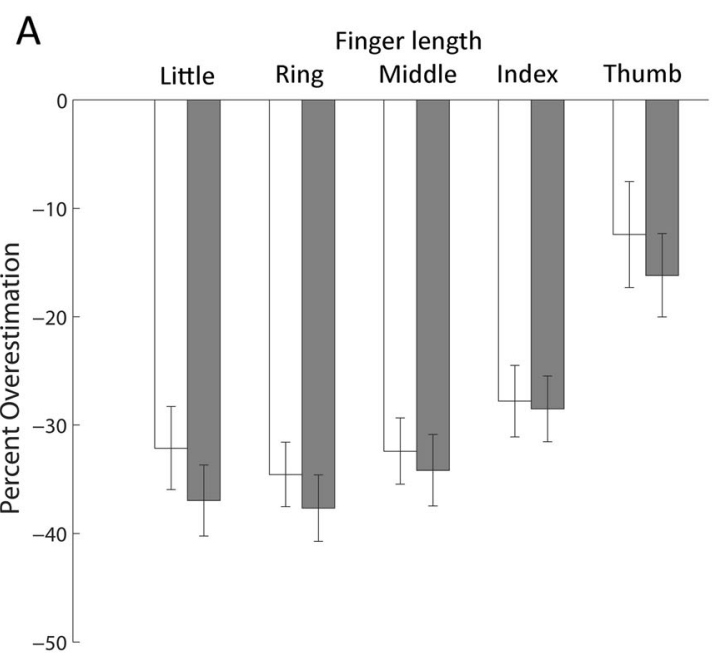

Fig. 1 Experiment 1 (a) percent overestimation [i.e., $100 \times$ (judged length - actual length)/actual length] of finger length. Clear underestimation was observed, in both apparent (white bars) and objective (black bars) conditions, increasing from radial (thumb) to the

from each other, $t(25)=1.29, p=.21, d_{\mathrm{z}}=0.25$. The generalised Procrustes superposition (GPS) of the configuration landmarks of each participant of their actual hand and the internal representation based on localization judgments in apparent and objective instruction conditions are depicted in Fig. 2.

The results from Experiment 1 revealed no apparent effects of instructions. The characteristic set of distortions found in previous studies was clearly apparent under both objective and apparent instructions. In particular, the magnitude of these distortions were similar to the ones found in previous reports both in terms of finger's length $(M=-35.9 \%$ : Longo, 2014; $M$ $=-27.9 \%$ : Longo \& Haggard, 2010; $M=-40.66 \%$ : Mattioni \& Longo, 2014) and hand width $(M=52.2 \%$ : Longo, 2014; $M=$ 67\%: Longo \& Haggard, 2010; $M=69 \%$ : Mattioni \& Longo,

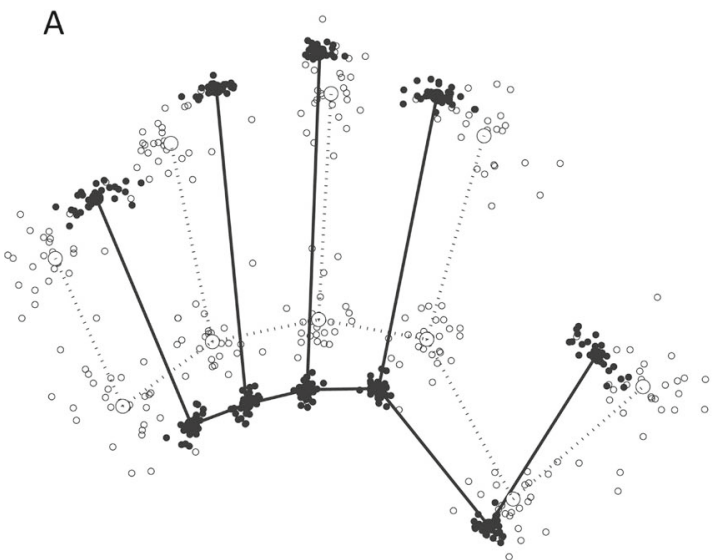

Apparent

Real hand

Fig. 2 GPS of landmark positions for actual hands (black full dots/black lines) and the body model inferred from the localization judgments in the (a) apparent (black empty dots/black dotted lines) and (b) objective (grey
B

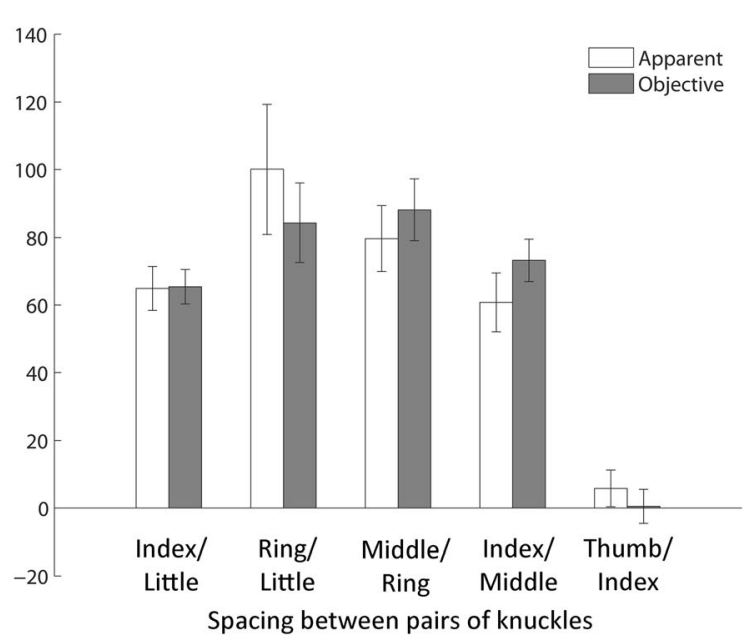

ulnar (little finger) side of the hand. Negative values indicate the degree of underestimation, and zero indicates correct reports. Error bars represent standard error of the mean $( \pm S E M)$. b Percentage overestimation of spacing between pairs of knuckles

2014) estimation, corroborating the suitability of our approach.

\section{Experiment 2: Tactile size perception along versus across the hand dorsum under apparent and objective instructions}

\section{Method}

Participants Twenty-six new participants (mean $\pm S D=25.0$ \pm 5.8 years; all females) took part in the study. Participants of all experiments reported normal or corrected-to-normal vision and normal touch. Participants were all right-handed except

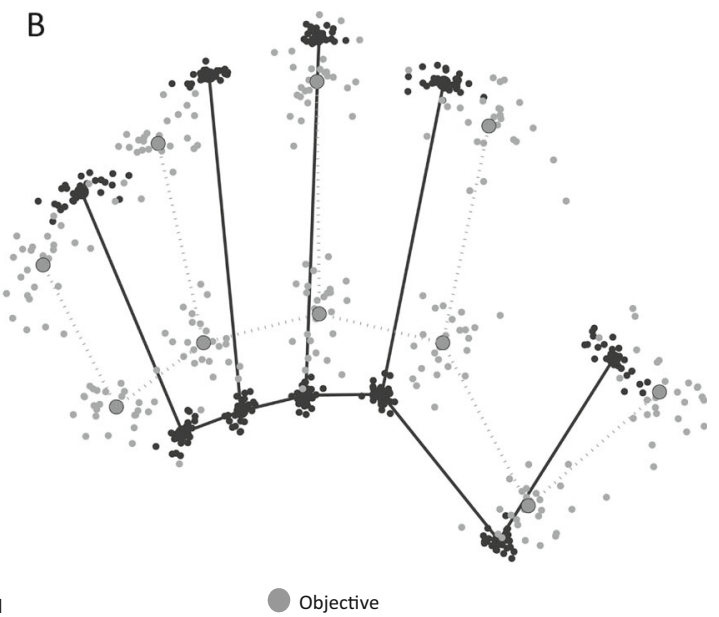

full dots/grey dotted lines) conditions, respectively. Solid line indicates mean shape of actual hand; dotted lines indicate mean shape of body model 
for one, as assessed by the Edinburgh Handedness Inventory (Oldfield, 1971; $M=81$, range: $-87-100$ ). One participant was discarded from the analysis because she reported feeling only a single point on most trials, even with the 4-cm stimulus (please see the Materials and Procedure sections about the tactile stimuli used).

Materials Stimuli were pairs of pointed wooden posts (diameter $1.5 \mathrm{~mm}$ ), mounted in foamboard and separated by $2,2.5$, $3,3.5$, or $4 \mathrm{~cm}$, similar to those used in previous studies (e.g., Longo \& Haggard, 2011). The tip of each rod tapered to a point, but was not sharp.

Procedure On each trial, participants were touched twice on the dorsum of their left hand, once with the posts oriented along the proximodistal axis of the hand (along stimulus) and once oriented along the mediolateral axis (across stimulus). Touch was applied approximately in the centre of the dorsum. Participants made untimed two-alternative forced choice (2AFC) judgments of whether the two points felt farther apart for the first or the second stimulus and responded verbally. As in Experiment 1, in different blocks of trials participants were asked to adopt different approaches to the task. At the beginning of the study, they were given the following instructions:

In different blocks of trials, I'll ask you to adopt different approaches to the task. On some blocks, I'll ask you to judge which of the two stimuli FEELS like it is bigger. In this case, we're interested in your subjective experience of the size of the stimuli. On other blocks, I'll ask you to judge which of the two stimuli you think REALLY IS bigger. In this case, we're interested in your objective beliefs about the size of the stimuli.

There were five tactile distances used: $2,2.5,3,3.5$, and $4 \mathrm{~cm}$. Every combination of the five stimuli as both the across and the along stimulus was used, counterbalancing the order of stimulus presentation across trials. Each of these stimulus pairs was repeated four times. This resulted in 100 trials per instruction condition (i.e., apparent vs. objective) presented in separate blocks of 50 trials each, for a total of 200 trials. The order of along and across stimuli was counterbalanced within each stimulus pair, and order of trials was randomized. The experimenter administered stimuli manually. The duration of each touch was approximately 1 second, with an interstimulus interval of approximately 1 second. Vision of the hand was prevented by means of a sheet of black cardboard throughout the procedure, and participants were not allowed to see the stimuli before testing commenced.

Analysis The proportion of trials in which the 'across' stimulus was judged as larger was analysed as a function of the ratio of the length of the along and across stimuli, plotted logarithmically to produce a symmetrical distribution about the pointof-actual-equality (i.e., ratio equals 1). Cumulative Gaussian functions were fit to each participant's data with least-squares regression using the Palamedes toolbox for MATLAB (Prins \& Kingdom, 2009). Points of subjective equality (PSEs) were determined as the point at which the psychometric function crossed $50 \%$. The slope of the psychometric function was quantified as the inverse of the standard deviation of the best fitting Gaussian.

\section{Results and discussion}

$R$-squared values for the psychometric functions of individual participants ranged from .276 to $.911(M \pm S E=.652 \pm .164)$ in the apparent condition and from .211 to $.864(M \pm S E=.621$ $\pm .038)$ in the objective condition, indicating comparable goodness of fit to the data for both type of instructions, $t(24)$ $=0.97, p<.34, d_{z}=0.19$.

Our main experimental question concerned the PSEs. If there is no distortion of hand shape, PSEs should, on average, equal 1 , indicating that stimulus orientation does not bias perceived size. If the hand is represented as being longer and more slender than it is, stimuli running across the hand would have to be larger than those running along the hand for the two to feel equivalent, and PSEs greater than 1 would be expected. Conversely, if the hand is represented as wider and more squat than it really is, stimuli running along the hand would have to be larger than those running across the hand for the two to feel equivalent, and PSEs less than 1 would be expected. Specifically, we were interested in testing whether these distortions may vary as a function of the type of instruction provided to the participants (apparent vs. objective).

As shown in Fig. 3, the mean PSE for the apparent $(M \pm S E$ $=.702 \pm .02)$ and objective $(M \pm S E=.725 \pm .02)$ instructions were significantly less than 1 in both cases, $t(24)=-10.79, p=$ $.0001, d=2.16$, and $t(24)=-9.64, p=.0001, d=1.97$, indicating a bias in representing the hand as wider than it really is. This is in agreement with previous reports showing that tactile stimuli running mediolaterally are systematically perceived as larger than stimuli running proximodistally (Longo \& Haggard, 2011). Moreover, distortions in hand shape were strongly correlated in both conditions, $r(24)=.725, p<$ .0001 . However, as in Experiment 1, we did not find a modulatory effect of the type of instruction on these distorted representations, $t(24)=-1.27, p=.22, d_{\mathrm{z}}=.26$. The mean slope for apparent $(M \pm S E \beta=6.8 \pm .74)$ was marginally significantly greater compared to objective $(M \pm S E \beta=5.6 \pm .44)$ instruction condition, $t(24)=2.01, p=.06, d_{\mathrm{z}}=0.40$.

These results replicate previous reports showing similar anisotropy on the hand dorsum (Canzoneri et al., 2013; Green, 1982; Le Cornu Knight, Longo, \& Bremner, 2014; Longo, Ghosh, \& Yahya, 2015; Longo \& Haggard, 2011; 


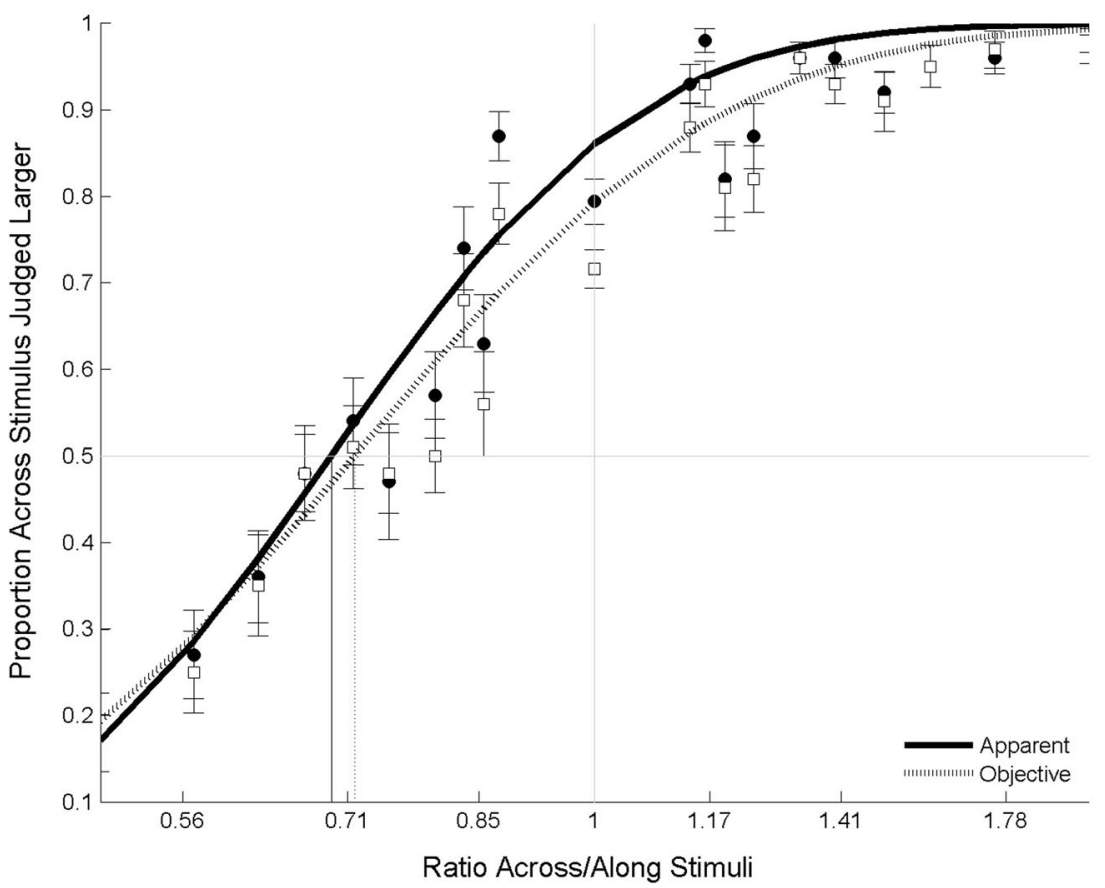

Fig. 3 Experiment 2 results in which curves are the cumulative Gaussian functions fit with least-squares regression. Vertical lines represent points of subjective equality (i.e., where the curve crosses $50 \%)$. Error bars represent the standard error of the mean $( \pm S E M)$

Longo \& Sadibolova, 2013; Miller, Longo, \& Saygin, 2014) with the presence of a clear bias for tactile distances to be perceived as larger when oriented mediolaterally, across the dorsum of the hand, than proximodistally, along the hand. Results of the present study match the PSE values of previous reports on the hand dorsum (PSE $=.740$ : Longo et al., 2015; Exp. 1, PSE = .758: Exp. 2, PSE = .739: Exp. 3, PSE = .719: Longo \& Haggard, 2011) and other body parts (Approximate PSE arm $=.5$, wrist $=.81$, and hand $=.8$; Le Cornu Knight et al., 2014). Moreover, our results show that this is a robust effect that is not modulated by the attitude that participants assume in performing the task (i.e., apparent vs. objective).

\section{Experiment 3: Explicit judgments of hand size under apparent and objective instructions}

\section{Method}

Participants The same participants as in Experiment 2 also completed this experiment. Half of the participants performed first Experiment 2, and half of them performed first Experiment 3.

Procedure In this metric body image task, participants have to adjust the length of a visually presented line on the screen. On each block, the participant was required to adjust the length of the line presented on screen to match the size of a part of their left hand. As in our previous study (Longo \& Haggard, 2012), there were six distances estimated: the length of each of the five fingers (i.e., the distance from the knuckle to the fingertip) and the distance between the knuckles of the index and little fingers as an overall measure of hand width. As for Experiments 1 and 2, participants were asked to adopt different approaches to the task. The following instructions were given at the beginning of the study, immediately after participants had completed Experiment 2:

As previously, in different blocks of trials, I'll ask you to adopt different approaches to the task. On some blocks, I'll ask you to adjust the length of the line so that it matches what it FEELS like the length of your body part is. In this case, we're interested in your subjective experience of the size of your body. On other blocks, I'll ask you to adjust the length of the line so that it matches what you think the length of your body part REALLY IS. In this case, we're interested in your objective beliefs about the size of your body.

There were four possible starting line conditions that occurred in a randomised order and differed by their orientation (horizontal, vertical) and starting length (small: 40 pixels/ $1.54 \mathrm{~cm}$; large: 460 pixels $/ 17.69 \mathrm{~cm}$ ). Lines were approximately $1-\mathrm{mm}$ thick and were white on a black background. Participants made unspeeded responses, adjusting the line length on the screen by pressing two arrow buttons on the keypad with the right hand. When they were satisfied by their estimation, they pressed an additional button to confirm their 
response. Vision of the hands during the task was prevented by means of a sheet of cardboard placed horizontally just above the hands.

There were 12 trials for each block of apparent and objective instruction conditions. Blocks were presented counterbalanced in ABBA sequence, and in each of them participants were randomly asked to match the size of the line with each of the six body parts (i.e., thumb, index, middle, ring, and index and little fingers knuckles) for a total of 48 trials. At the end of the experiment, a photograph was taken of the participant's left hand to calculate the actual size of each body part. A ruler appeared in the photographs, allowing conversion between distances in pixels and $\mathrm{cm}$. As in Experiment 1, a black dot was made with a felt pen on the knuckle of each finger to facilitate coding.

\section{Results and discussion}

As shown in Fig. 4, there was clear underestimation of finger length, which increased across the hand. Collapsing the five fingers, there was significant underestimation of finger length for both the apparent $(M \pm S E=-11.3 \% \pm 3.6), t(25)=-3.20, p$ $=.004, d=0.63$, and objective $(M \pm S E=-12.8 \% \pm 3.4), t(25)$ $=-3.73, p=.001, d=0.73$, instruction conditions. As in Experiments 1 and 2, we found no significant difference between the different instruction conditions, $t(25)=0.76, p=$ $.46, d_{\mathrm{z}}=0.15$. There was a significant correlation of the fingers length underestimation in the two conditions, $r(25)=$ $.871, p=.0001$.
Underestimation increased along a radial-ulnar gradient for both the apparent $(M \pm S E \beta=7.2 \% \pm .97 /$ finger $), t(25)$ $=-7.42, p<.0001, d=1.46$, and objective $(M \pm S E \beta=6.6 \% \pm$ $.79 /$ finger $), t(25)=-8.35, p<.0001, d=1.64$, conditions. The magnitude of this gradient did not differ between the apparent and objective instructions, $t(25)=-0.80, p=.43, d_{\mathrm{z}}=0.16$. There was a significant correlation between slopes in the two conditions, $r(25)=.621, p=.001$. As for the previous experiments, the type of instruction provided to the participants did not affect the distorted representation of the fingers length.

Consistent with Experiment 1, there was overestimation of hand width for both the apparent $(M \pm S E=36.2 \% \pm 7.1)$, $t(25)=5.10, p<.0001, d=1.00$, and objective $(M \pm S E=$ $29.1 \% \pm 5.6), t(25)=5.18, p<.0001, d=1.02$, instructions. The magnitude of overestimation did not differ between the two conditions, $t(25)=1.54, p=.14, d_{\mathrm{z}}=0.30$. There was a significant correlation between the magnitude of overestimation in the two conditions (i.e., apparent and objective) for the hand width measure, $r(25)=.763, p<0.0001$.

The shape index used in Experiment 1 can also be calculated for the line length task, allowing comparison of overall hand shape across the tasks as well as the actual hand. As for Experiment 1, shape indices were greater than for the actual hand for both the apparent (92.3 vs, 56.7), $t(25)=-8.78, p<$ $.0001, d=1.72$, and objective (89.1 vs. 56.7$), t(25)=-8.48, p$ $<.0001, d=1.66$, instructions. The shape index for the two experimental conditions did not differ significantly from each other, $t(25)=1.00, p=.33, d_{\mathrm{z}}=0.20$. As previously, the

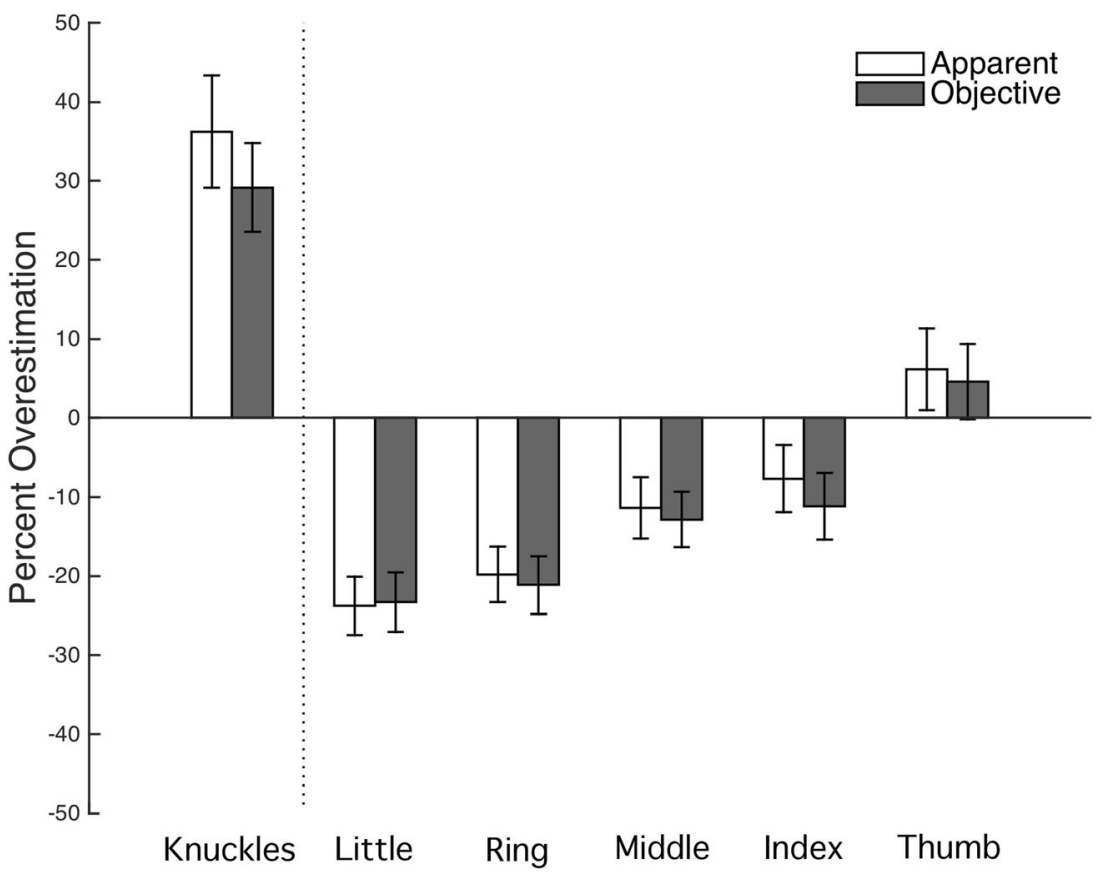

Fig. 4 Results from Experiment 3, showing percentage overestimation [i.e., $100 \times$ (judged length - actual length)/actual length] of finger length and knuckle spacing for the judgment of the line length task. Clear underestimation of the finger length was observed, which increased

progressively from the thumb to the little finger. Conversely, there was clear overestimation of hand width. Error bars represent the standard error of the mean $( \pm S E M)$ 
pattern of results is compatible with earlier reports using the same paradigm. For instance, Longo and Haggard (2012) found underestimation of fingers' length $(M=-23 \%)$ with an increment along a radial-ulnar gradient $(M \beta=3.2 \%$; Longo \& Haggard, 2012).

\section{Between Experiments (1, 2, 3) analyses on first experimental block}

One possible concern about these results is that because participants were asked to go back and forth between different types of judgments, they might have attempted to respond consistently across all blocks rather than following the instructions. To control for the possibility, considering that we have used a within-subject design where the block were presented in an ABBA order, we analysed the influence of condition by comparing only the first experimental block for each participant. For this block, the participant had only been asked to make a single type of response, so participants would have not made prior estimates with which they were trying to be consistent. To this end, values of fingers' length percentage overestimation of the first block for Experiment 1 were entered into an ANOVA with FINGER LENGTH (thumb, index, middle, ring, little) as within-participants factor and INSTRUCTION (apparent, objective) as between-participants factor. As expected, the analysis revealed a main effect of FINGER LENGTH, $F(4$, $100)=28.622, p=.0001, M S E=287, \eta_{\mathrm{p}}{ }^{2}=.53$; however, there was no main effect of instruction, $F(1,25)=2.45, p=$ $.13, M S E=459, \eta_{\mathrm{p}}{ }^{2}=.089$, nor significant interactions (all $p \mathrm{~s}$ $>$.44). Similarly, for Experiment 1 we entered the values of the percentage overestimation of participants' hand width of the first block into an ANOVA with HAND WIDTH (thumb/index, index/middle, middle/ring, ring/little, index/little) as within-participants factor and INSTRUCTION (apparent, objective) as between-participants factor. Analysis revealed a main effect of HAND WIDTH, $F(4,100)=24.289, p=.0001, M S E=$ $5,278, \eta_{\mathrm{p}}{ }^{2}=.50$, but no significant main effect of INSTRUCTION nor interactions (all $p \mathrm{~s}>.13$ ). For Experiment 2, the PSE values for the first block were entered into a one-way ANOVA with INSTRUCTION (apparent, objective) as betweenparticipants factor. This analysis did not revealed a significant main effect of INSTRUCTION, $F(1,24)=0.544, p=.47, M S E=$ $0.20, \eta_{\mathrm{p}}{ }^{2}=.02$. Finally, values of fingers' length percentage overestimation of the first block for Experiment 3 were entered into an ANOVA with FINGER LENGTH (thumb, index, middle, ring, little) as within-participants factor and INSTRUCTION (apparent, objective) as between-participants factor. Also, this analysis revealed a main effect of FINGER LENGTH, $F(5,120)=28.372, p=.0001, M S E=0.04, \eta_{\mathrm{p}}{ }^{2}=$ .54 ; however, there was no main effect of instruction, $F(1,24)$ $=2.090, p=.85, M S E=0.22, \eta_{\mathrm{p}}{ }^{2}=.08$, nor significant interactions, $F(5,120)=2.045, p=.08, M S E=0.04, \eta_{\mathrm{p}}{ }^{2}=.08$. Overall, these analyses show that in all experiments, using a between subjects analysis, there were no differences between apparent and objective instruction conditions. This suggests that the lack of an effect in the full analyses is unlikely to derive from participants attempting to be consistent in their judgements.

\section{General discussion}

Similar distortions of hand size and shape were found whether participants were asked to localise landmarks indicated by verbal labels (Experiment 1: position sense), asked to make 2AFC judgments of which distance felt larger on pairs of touches presented in different orientations on the dorsum of their hand (Experiment 2: tactile size perception), or adjust the length of lines presented on a screen to match the length of different parts of their hand (Experiment 3: conscious body image). Critically, there were no differences between apparent and objective instructions. In agreement with previous findings, three types of distortions of the implicit hand maps emerged in the two instruction conditions. These include underestimation of finger length (i.e., distance between the knuckles and tip of the fingers), overestimation of the hand width (i.e., distance between pairs of knuckles), and a radialulnar gradient with underestimation of finger length increasing systematically from the thumb to the little finger. These results demonstrate that the distortions revealed by these tasks are robust to different types of instructions and suggest that the distortions do not reflect a dissociation between participants' subjective experiences and actual beliefs.

These findings have strong implications for understanding the origins of the distortions we have observed. In particular, one critical point is whether these misrepresentations are really distortions of the body or instead can derive, at least to some extent, from the specific response attitude that the type of instructions (i.e., apparent vs. objective) given to the participants generates. Indeed, asking participants to give judgments about their subjective feelings (i.e., apparent instructions) of stimuli compared to asking them to give judgements about their actual beliefs (i.e., objective instructions) about stimuli have been shown to significantly affect behavioural performance across a wide range of tasks (Gogel \& Da Silva, 1987; Leibowitz \& Harvey, 1969; Wagner, 2006).

In particular, a previous study that used an approach similar to ours (Ekroll et al., 2016) found that estimation of finger length was differently modulated depending on the approach used, with greater effects of the 'shrunken finger illusion' for apparent than objective instructions. However, in their study, authors found, though less, some distortions also in the objective condition, suggesting that participants were not completely able to distinguish between their actual beliefs and their subjective feelings (Ekroll et al., 2016). This result is important in demonstrating that participants are able to discriminate 
the difference between the two instruction types in terms of making judgments about the body. The findings of Ekroll and colleagues fit with the fact that participants experiencing the Pinocchio illusion (Lackner, 1988) do not really believe that their nose is lengthening, and those experiencing the rubber hand illusion (Botvinick \& Cohen, 1998) do not really believe the rubber hand is part of their body. Thus, the rapid plasticity of the body image seen in these and other body illusions affects subjective feelings, not actual beliefs-apparent rather than objective judgments. In contrast, these results reveal no such dissociation in terms of baseline distortions. This suggests that the distortions reported to underlie position sense (Longo \& Haggard, 2010), tactile distance perception (Longo \& Haggard, 2011), and the body image (Longo \& Haggard, 2012) are fundamentally different from illusions which modulate body perception.

These different response approaches are thought to be mediated by different types of processing. Namely, apparent instructions seem to direct observers to base their judgments on more perceptual sources of spatial information, whereas objective instructions seem to direct observers to rely on more cognitive sources of spatial information (Predebon, 1992). The present results demonstrate that the distortions of body representations we have previously described are not a result of the specific attitude used by the participants to respond, even when, as in this series of experiments, bias instructions are emphasized on purpose. The fact that participants using both 'apparent' and 'objective' instructions in the present work is consistent with results of previous studies in which only 'apparent' instructions were used (e.g., Longo \& Haggard, 2010, 2011, 2012) suggests that misrepresentations do not result from a dissociation between subjective feelings and actual beliefs. That is, these distortions are not like cases such as the Aristotle illusion (Benedetti, 1985), the rubber hand illusion (Botvinick \& Cohen, 1998), the shrunken finger illusion (Ekroll et al., 2016), or phantom limbs (Melzack, 1990), in which people's feelings about their body do not correspond with their actual beliefs.

Similarly, the same specific distortions of the hand are present across a wide variety of tasks spanning several sensory modalities. Distorted maps of the hand are present also when participants have both visual and proprioceptive feedback about the location of their responses (Longo \& Haggard, 2010), when they have only proprioceptive feedback (Longo, 2014), and when they have only visual feedback (Longo et al., 2012). In addition, distortions persist even when individuals estimate the dimensions of their hands under magnification (Linkenauger, Geuss, et al., 2014). Furthermore, different distortions have been reported for judgments of the two sides of the hand (i.e., palm and dorsum surfaces) using identical instructions in the two cases. In the same vein, distorted perception of the relative dimensions of the whole body persists even when the participant is asked to made their estimates while standing in front of a mirror, which provides full, unambiguous visual information specifying the relative dimensions of their body (Linkenauger, Wong, et al., 2014). Moreover, Cardinali and colleagues (2009), in a series of experiments, investigated the perceived length of the upper limb (i.e., arm and hand). Participants were asked to estimate the distance between two tactually stimulated points on the skin, before and after a grasping training using a mechanical grabber. They found that after tool-use, participants tend to localise the positions of the touches on the elbow and fingertip as being farther apart (Cardinali et al., 2009). Similarly, Sposito and colleagues (2012), using a line-bisection task, asked participants to estimate the middle point of their forearm before and after a training using a $60-\mathrm{cm}$ tool. After the training, participants estimated the point to be more distal, which the authors interpreted as evidence of an increase in the perceived length of the arm (Sposito, Bolognini, Vallar, \& Maravita, 2012). Overall, these results suggest that these distortions reflect representations of the body itself, rather than specific response attitudes that participants decide to adopt or that are unintentionally generated by the experimenter.

Recently, in the psychological sciences, there has been increasing awareness of the importance of replication due to mounting frustration among researchers failing to replicate important findings in the field. As a consequence, the obligation to conduct direct and conceptual replications of one's work has never been stronger to protect ourselves from basing our theoretical foundations on unreliable findings. This need is certainly stronger for surprising findings that defy our natural intuitions, as those are the findings that researchers have had the most difficulty in replicating (Hartshorne \& Schachner, 2012). The finding that the most familiar part of our body, the hand, is perceived as drastically distorted (on average $40 \%$ ) is certainly surprising. We have an abundance of visual information to directly specify the exact relative dimensions of our hands, and we, arguably, look at our hands more than we look at anything else. True distortions of this size have substantial implications for understanding how the human body is perceived. Hence, we recognize the need to determine whether or not these distortions are real or merely a by-product of task demands or inadvertent experimenter bias. Here, we all but told participants that we expected them to behave differently in each instruction condition, and the same distortions were found in both conditions to a similar magnitude. Our results suggest that body distortions are not susceptible to the type of instructions (i.e., apparent or objective) given to the participants and are robust across various task demands.

Acknowledgements This research was supported by a grant from the European Research Council (ERC-2013-StG-336050) under the FP7 to M.R.L. 


\section{References}

Benedetti, F. (1985). Processing of tactile spatial information with crossed fingers. Journal of Experimental Psychology: Human Perception and Performance, 11(4), 517-525.

Ben-Tovim, D. I., Walker, M. K., Murray, H., \& Chin, G. (1990). Body size estimates: Body image or body attitude measures? International Journal of Eating Disorders, 9(1), 57-67.

Bookstein, F. (1991). Morphometric tools for landmark data: Geometry and biology. Cambridge, UK: Cambridge University Press.

Boring, E. G. (1921). The stimulus-error. The American Journal of Psychology, 32(4), 449. doi:10.2307/1413768

Botvinick, M., \& Cohen, J. (1998). Rubber hands "feel" touch that eyes see. Nature, 391(6669), 756. doi:10.1038/35784

Brugger, P., Lenggenhager, B., \& Giummarra, M. J. (2013). Xenomelia: A social neuroscience view of altered bodily self-consciousness. Frontiers in Psychology, 4, 204. doi:10.3389/fpsyg.2013.00204

Canzoneri, E., Ubaldi, S., Rastelli, V., Finisguerra, A., Bassolino, M., \& Serino, A. (2013). Tool-use reshapes the boundaries of body and peripersonal space representations. Experimental Brain Research, 228(1), 25-42. doi:10.1007/s00221-013-3532-2

Cardinali, L., Frassinetti, F., Brozzoli, C., Urquizar, C., Roy, A. C., \& Farnè, A. (2009). Tool-use induces morphological updating of the body schema. Current Biology: CB, 19(12), R478-R479. doi:10. 1016/j.cub.2009.05.009

Carlson, V. (1977). Instructions and perceptual constancy judgments. In W. Epstein (Ed.), Stability and constancy in visual perception: Mechanisms and processes (pp. 217-254). New York, NY: Wiley.

Cash, T. F., \& Deagle, E. A. (1997). The nature and extent of body-image disturbances in anorexia nervosa and bulimia nervosa: A meta-analysis. The International Journal of Eating Disorders, 22(2), 107-125.

Chirimuuta, M. (2016). Why the "stimulus-error" did not go away. Studies in History and Philosophy of Science Part A, 56, 33-42. doi:10.1016/j.shpsa.2015.10.007

Ekroll, V., Sayim, B., Van der Hallen, R., \& Wagemans, J. (2016). Illusory visual completion of an object's invisible backside can make your finger feel shorter. Current Biology: CB, 26(8), 10291033. doi:10.1016/j.cub.2016.02.001

Ferrè, E. R., Vagnoni, E., \& Haggard, P. (2013). Vestibular contributions to bodily awareness. Neuropsychologia, 51(8), 1445-1452. doi:10. 1016/j.neuropsychologia.2013.04.006

Fuentes, C. T., Longo, M. R., \& Haggard, P. (2013). Body image distortions in healthy adults. Acta Psychologica, 144(2), 344-351. doi:10. 1016/j.actpsy.2013.06.012

Gogel, W. C., \& Da Silva, J. A. (1987). Familiar size and the theory of off-sized perceptions. Perception \& Psychophysics, 41(4), 318-328.

Gower, J. (1975). Generalized procrustes analysis. Psychometrika, 40(1), $33-51$.

Green, B. G. (1982). The perception of distance and location for dual tactile pressures. Perception \& Psychophysics, 31(4), 315-323.

Hach, S., \& Schütz-Bosbach, S. (2010). Sinistrals' upper hand: Evidence for handedness differences in the representation of body space. Brain and Cognition, 72(3), 408-418. doi:10.1016/j.bandc.2009.12.001

Hartshorne, J. K., \& Schachner, A. (2012). Tracking replicability as a method of post-publication open evaluation. Frontiers in Computational Neuroscience, 6, 8. doi:10.3389/fncom.2012.00008

Lackner, J. R. (1988). Some proprioceptive influences on the perceptual representation of body shape and orientation. Brain, 111(Pt 2), 281297.

Le Cornu Knight, F., Longo, M. R., \& Bremner, A. J. (2014). Categorical perception of tactile distance. Cognition, 131(2), 254-262.

Leibowitz, H. W., \& Harvey, L. O. (1969). Effect of instructions, environment, and type of test object on matched size. Journal of Experimental Psychology, 81(1), 36-43.
Linkenauger, S. A., Geuss, M. N., Stefanucci, J. K., Leyrer, M., Richardson, B. H., Proffitt, D. R., . . . Mohler, B. J. (2014). Evidence for hand-size constancy: The dominant hand as a natural perceptual metric. Psychological Science, 25(11), 2086-2094. doi: 10.1177/0956797614548875

Linkenauger, S. A., Witt, J. K., Bakdash, J. Z., Stefanucci, J. K., \& Proffitt, D. R. (2009). Asymmetrical body perception: A possible role for neural body representations. Psychological Science, 20(11), 1373-1380. doi:10.1111/j.1467-9280.2009.02447.x

Linkenauger, S. A., Wong, H. Y., Geuss, M., Stefanucci, J. K., McCulloch, K. C., Bülthoff, H. H., . . . Proffitt, D. R. (2014). The perceptual homunculus: The perception of the relative proportions of the human body. Journal of Experimental Psychology: General. doi: $10.1037 /$ xge0000028

Longo, M. R. (2014). The effects of immediate vision on implicit hand maps. Experimental Brain Research, 232(4), 1241-1247. doi:10. 1007/s00221-014-3840-1

Longo, M. R., Azañón, E., \& Haggard, P. (2010). More than skin deep: Body representation beyond primary somatosensory cortex. Neuropsychologia, 48(3), 655-668.

Longo, M. R., Ghosh, A., \& Yahya, T. (2015). Bilateral symmetry of distortions of tactile size perception. Perception, 44(11), 12511262. doi:10.1177/0301006615594949

Longo, M. R., \& Haggard, P. (2010). An implicit body representation underlying human position sense. Proceedings of the National Academy of Sciences of the United States of America, 107(26), 11727-11732. doi:10.1073/pnas.1003483107

Longo, M. R., \& Haggard, P. (2011). Weber's illusion and body shape: Anisotropy of tactile size perception on the hand. Journal of Experimental Psychology. Human Perception and Performance, 37(3), 720-726.

Longo, M. R., \& Haggard, P. (2012). Implicit body representations and the conscious body image. Acta Psychologica, 141(2), 164-168.

Longo, M. R., Long, C., \& Haggard, P. (2012). Mapping the invisible hand: A body model of a phantom limb. Psychological Science, 23(7), 740-742. doi:10.1177/0956797612441219

Longo, M. R., \& Sadibolova, R. (2013). Seeing the body distorts tactile size perception. Cognition, 126(3), 475-481. doi:10.1016/j. cognition.2012.11.013

Lopez, C., Schreyer, H. M., Preuss, N., \& Mast, F. W. (2012). Vestibular stimulation modifies the body schema. Neuropsychologia, 50(8), 1830-1837. doi:10.1016/j.neuropsychologia.2012.04.008

Mattioni, S., \& Longo, M. R. (2014). The effects of verbal cueing on implicit hand maps. Acta Psychologica, 153, 60-65. doi:10.1016/j. actpsy.2014.09.009

Melzack, R. (1990). Phantom limbs and the concept of a neuromatrix. Trends in Neurosciences, 13(3), 88-92.

Miller, L. E., Longo, M. R., \& Saygin, A. P. (2014). Tool morphology constrains the effects of tool use on body representations. Journal of Experimental Psychology. Human Perception and Performance, 40(6), 2143-2153. doi:10.1037/a0037777

Napier, J. (1980). Hands. Princeton, NJ: Princeton University Press.

Oldfield, R. C. (1971). The assessment and analysis of handedness: The Edinburgh inventory. Neuropsychologia, 9(1), 97-113.

Phillips, K. A. (2005). The broken mirror: Understanding and treating body dysmorphic disorder (2nd ed.). Oxford, UK: Oxford University Press.

Predebon, J. (1980). Length judgments of body parts. Perceptual and Motor Skills, 51(1), 83-88.

Predebon, J. (1992). The role of instructions and familiar size in absolute judgments of size and distance. Perception \& Psychophysics, 51(4), 344-354.

Prins, N., \& Kingdom, F. (2009). Palamedes: Matlab routines for analyzing psychophysical data. Retrieved from http://www.palamedestoolbox. org 
Proske, U., \& Gandevia, S. C. (2012). The proprioceptive senses: Their roles in signaling body shape, body position and movement, and muscle force. Physiological Reviews, 92(4), 1651-1697. doi:10. 1152/physrev.00048.2011

Ramachandran, V. S., \& Hirstein, W. (1998). The perception of phantom limbs. The D. O. Hebb lecture. Brain: A Journal of Neurology, 121(Pt. 9), 1603-1630.

Saulton, A., Dodds, T. J., Bülthoff, H. H., \& de la Rosa, S. (2015). Objects exhibit body model like shape distortions. Experimental Brain Research. doi:10.1007/s00221-015-4221-0

Slade, P. D., \& Russell, G. F. (1973). Awareness of body dimensions in anorexia nervosa: Cross-sectional and longitudinal studies. Psychological Medicine, 3(2), 188-199.

Sposito, A., Bolognini, N., Vallar, G., \& Maravita, A. (2012). Extension of perceived arm length following tool-use: Clues to plasticity of body metrics. Neuropsychologia, 50(9), 2187-2194. doi:10.1016/j. neuropsychologia.2012.05.022

Ten Berge, J. M. F. (1977). Orthogonal procrustes rotation for two or more matrices. Psychometrika, 42(2), 267-276.

Titchener, E. (1909). Lectures on the experimental psychology of the thought-processes. New York, NY: Macmillan.

Treasure, J., Claudino, A. M., \& Zucker, N. (2010). Eating disorders. Lancet, 375(9714), 583-593. doi:10.1016/S0140-6736(09)61748-7

Vallar, G., \& Ronchi, R. (2009). Somatoparaphrenia: A body delusion. A review of the neuropsychological literature. Experimental Brain Research, 192(3), 533-551. doi:10.1007/s00221-008-1562-y

Wagner, M. (2006). The geometries of visual space. Mahwah, NJ: Erlbaum. Weber, E. H. (1996). De subtilitate tactus. In H. Ross \& D. Murray (Eds.), E. H. Weber on the tactile senses (2nd ed., pp. 21-128). London, UK: Academic Press. Original work published 1834. 